\title{
Original
}

\section{Laparo-endoscopia por acceso único: experiencia inicial}

\author{
Rene Sotelo, Juan Carlos Astigueta, Oswaldo Carmona, Robert De Andrade, Rafael Sanchez-Salas \\ Centro de Cirugía Robótica y Mínimamente Invasiva. Unidad de Urología. \\ Instituto Médico La Floresta, Caracas-Venezuela
}

\section{Resumen}

Objetivo: Presentar nuestra experiencia inicial en LESS Surgery (Laparo-Endoscopic Single Site Surgery/Cirugía laparoendoscópica por acceso único), mediante uso de dispositivo multicanal, instrumentos estándar, articulables y otros adaptados para la consecución de los procedimientos.

Materiales y metodos: Entre febrero y septiembre del 2008 se realizaron 28 procedimientos quirúrgicos LESS con puerto multicanal: prostatectomía simple transumbilical (PSTU) y transvesical (PSTV), nefrectomía simple (NS), enterocistoplastia de aumento (ECA) e histerectomía simple (HS). Los datos clínicos fueron recopilados de manera prospectiva y analizados retrospectivamente. Se utilizó dispositivo de acceso multicanal (R-Port). Los procedimientos fueron realizados en un centro por un solo cirujano (RS). La técnica quirúrgica empleada correspondió a la misma utilizada por vía laparoscópica convencional.

Resultados: Se realizaron 28 intervenciones quirúrgicas: PSTU(01), PSTV(20), NS(01), ECA(01), HS(05). La incisión de acceso fue realizada a nivel umbilical o infraumbilical. Solo un caso (nefrectomía) ameritó uso de trocar adicional de $2 \mathrm{~mm}$. La edad media en años por procedimiento fue: PSTU, 67; PSTV, 68 (57-89); NS, 12; ECA, 20; HS, 46.4 (41-54). El tiempo quirúrgico medio en minutos fue: PSTU, 120; PSTV, 91 (45-210); NS, 120; ECA, 300; HS, 112 (90-160). El sangrado operatorio fue: PSTU, 200cc; PSTV, 337 cc (50-1500); NS, 100cc; EAC, 100cc; HS, 118cc. (100-160). La complicación observada fue hematuria profusa en dos casos de PSTV, ambos pacientes requirieron exploración postoperatoria con evolución satisfactoria.

Conclusiones: LESS es una alternativa factible y reproducible en patología uroginecológica de resolución quirúrgica. Mayores estudios, experiencias y seguimiento permitirán la evaluación objetiva de esta técnica.

Palabras clave: LESS. Prostatectomía simple. Nefrectomía. Histerectomía. Enterocistoplastia.

\section{Laparo-endoscopic single site (LESS)}

\section{Abstract}

Objetive: We present our initial experience in laparo-endoscopic single site (LESS) surgery, through multichannel port, articulated instruments and standard instruments adapted others adapted for the procedures.

Materials and methods: Between February and October 2008, 28 LESS surgical procedures with multi-port were performed (Transumbilical simple prostatectomy (PSTU) and transvesical (PSTV), simple nephrectomy (NS), Enterocystoplasty augmentation (ECA) and simple hysterectomy (HS)). The clinical data were collected prospectively and analyzed retrospectively. Was used access device Multichannel (R-Port). The procedures were performed for a single surgeon (RS). The surgical technique was the same route used by conventional laparoscopic.

Results: Twenty-eight surgeries were performed: PSTU (01), PSTV (20), NS (01), ECA (01), HS (05). The incision was performed umbilical or infraumbilical. Only one case (nephrectomy) needed it placement of an additional trocar. The average age by procedure was PSTU and PSTV, 67 and 68 year-old (57-89 y) respectively; NS 12 year-old; RCT 20 year-old; HS 46.4 year-old (41 - 54 y). The operative time was: PSTU 120 minutes, PSTV 91 minutes (45-210 min); NS 120 minutes; RCT 300 minutes; HS 112 minutes(90-160 min). The operative bleeding was PSTU and PSTV $200 \mathrm{cc}$. and $337 \mathrm{cc}$ (50-1500 cc) respectively; NS 100cc; EAC $100 \mathrm{cc}$; HS $118 \mathrm{cc}$ (100-160 cc). The complication was haematury in two cases of PSTV, both patients required exploration postoperative endoscopic with satisfactory evolution.

Conclusions: LESS is a feasible and reproducible surgical option in uro-gynecologic surgical treatment. Further studies, experience and follow-up will provide an objective assessment of the technique.

Keywords: LESS. Simple prostatectomy. Nephrectomy. Hysterectomy. Enterocystoplasty. 
$\mathrm{E}$ la evolución de las terapias mínimamente invasivas se desarrolló la cirugía de NOTES (Natural Orifice Translumenal Endoscopic Surgery), con la finalidad de ejecutar procedimientos quirúrgicos sin trauma de la pared abdominal, a través de orificios naturales ${ }^{1,2}$.Sin embargo, debido a dificultades técnicas no se logró una adecuada difusión y se ha cambiado el enfoque de realización de esta técnica quirúrgica, surgiendo alternativas intermedias o hibridas, realizando una sola incisión en la cicatriz umbilical a través de la cual se colocan varios trocares o un trocar con múltiples canales ${ }^{3,7}$ Esta nueva modalidad a recibido diferentes denominaciones como E-NOTES (Embryonic Natural Orifice Trans Umbilical Endoscopic Surgery), NOTUS (Natural Orifice Trans Umbilical Surgery) y SPA (Single Port Access), entre otros ${ }^{8-12}$. Finalmente, en la reciente reunión del Consorcio de SinglePort, se llega al acuerdo del uso del término LESS (Laparo-Endoscopic Single Site Surgery), para nombrar todos los procedimientos laparoscópicos o endoscópicos realizados a través de una sola incisión abdominal ${ }^{13-14}$.El desarrollo de cirugía LESS en el campo de la Urología ha sido vertiginoso, especialmente a partir del año 2007, reportándose múltiples experiencias clínicas (Tabla 1).

En esta continua evolución y con la disponibilidad de equipos endoscópicos de menor calibre, articulables y de mejor calidad óptica, además del desarrollo de instrumentos articulables, se reinició la exploración y ejecución de diferentes cirugías con la técnica LESS. Las primeras experiencias se llevaron a cabo, con la colocación varios trocares juntos en una incisión única en la cicatriz umbilical, con la expectativa de que los instrumentos articulados permitiesen mantener el principio de la triangulación; posteriormente se optimizó el perfil de los trocares disminuyendo el posible conflicto externo entre ellos y finalmente se procedió al empleo de dispositivos con múltiples canales. Actualmente existen múltiples esfuerzos en la industria, en el desarrollo del dispositivo multicanal "ideal": que requiera una herida de mínima longitud, y que además permita el ingreso y salida de los diversos instrumentos articulados o doblados, hermético, reusable y de costo accesible. Ya existen en el mercado algunos de estos dispositivos, sin embargo cada día se sigue trabajando en el proceso de mejoramiento de prototipos, aun no existiendo el trocar perfecto. Con el uso de estos dispositivos, se han reportado dife- rentes cirugías como apendicetomía, colecistectomía, prostatectomía radical, cirugía retroperitoneal, histerectomía, cistectomía radical, pieloplastía, nefrectomía y hemicolectomía entre otros ${ }^{15,27}$. Presentamos nuestra experiencia quirúrgica inicial en cirugía LESS utilizando el dispositivo multicanal R-Port.

\section{MATERIAL Y METODOS}

Entre febrero y septiembre del año 2008, utilizando un dispositivo multicanal se han realizado 28 intervenciones quirúrgicas: prostatectomía simple transumbilical $(01)$, prostatectomía simple transvesical (20), nefrectomía simple (01), enterocistoplastia de aumento (01), e histerectomía simple (05), estas últimas en coordinación con el Servicio de Ginecología de nuestra institución. Los datos clínicos fueron recopilados de manera prospectiva $\mathrm{y}$ analizados retrospectivamente. Los procedimientos fueron realizados en un centro por un solo cirujano (RS).

La técnica quirúrgica empleada correspondió a la misma utilizada por vía laparoscópica convencional. En todos los casos se utilizo el dispositivo R-Port (Advanced Surgical Concepts, Dublin, Ireland).

Existen en la actualidad dos modelos disponibles de R-Port, que difieren en el diámetro y el número de canales (TriPort o QuadPort). Ambos modelos constan de un retractor y una válvula. El retractor se compone de un anillo interior, dos anillos externos y una manga de plástico retráctil. La válvula dependiendo del modelo presenta tres orificios (dos de $5 \mathrm{~mm}$ y uno de $12 \mathrm{~mm}$ ) o cuatro orificios (todos de $12 \mathrm{~mm}$ ), además de un puerto para insuflación en ambos modelos y otro de salida en el QuadPort. Los orificios de la válvula contienen un elastómero termoplástico que permite la correcta introducción de los instrumentos, incluidos agujas, con insignificante pérdida de $\mathrm{CO} 2$ (Fig 1).

Hasta fines del primer semestre del presente año

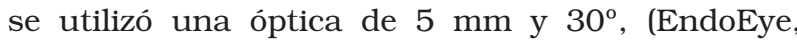
Olympus Medical, Tokyo, Japan), para luego dar paso a la óptica de $5 \mathrm{~mm}$ de punta flexible (EndoEye, Olympus Medical, Tokyo, Japan), la utilización de esta última, ha permitido trabajar con mayor comodidad en un espacio quirúrgico reducido, ya que no es necesario se mantenga en el mismo eje de los instrumentos del cirujano permitiéndole más libertad en sus movimientos y mejor ángulo de visión. Además, se utilizaron instrumentos flexibles 
Tabla 1. Experiencia clínica en cirugía LESS

\begin{tabular}{|c|c|c|c|c|c|c|}
\hline Autores & $\begin{array}{l}\text { Procedimiento } \\
\text { Urológico y número }\end{array}$ & $\begin{array}{l}\text { Tiempo } \\
\text { Quirúrgico } \\
\text { (horas) }\end{array}$ & $\begin{array}{l}\text { Sangrado } \\
\text { (cc) }\end{array}$ & $\begin{array}{l}\text { Hospitalización } \\
\text { (dias) }\end{array}$ & Acceso & Complicaciones \\
\hline $\begin{array}{l}\text { Kaouk et al, } \\
\text { J Urol, } 2008\end{array}$ & $\begin{array}{l}\text { Crioterapia retroperitoneal (4), } \\
\text { crioterapia transperitoneal (2), } \\
\text { biopsia renal en cuña (1), } \\
\text { nefrectomía simple (2), } \\
\text { nefrectomía radical (1) }\end{array}$ & 2.8 & 90 & 2.8 & Uni-X & Transfusión \\
\hline $\begin{array}{l}\text { Kaouk et al, } \\
\text { J Urol, } 2008\end{array}$ & Sacro-colpopexia (5) & 2.5 & 90 & 2 & Uni-X & Ninguna \\
\hline $\begin{array}{l}\text { Rane et al, } \\
\text { J Endourol, } \\
2007\end{array}$ & $\begin{array}{l}\text { Nefrectomia simple (2), } \\
\text { orquiopexia(1), } \\
\text { orquiectomia(1), } \\
\text { ureterolitotomia (1) }\end{array}$ & 1.3 & NP & NP & R-Port & Ninguna \\
\hline $\begin{array}{l}\text { Desai et al, } \\
\text { J Urol, } 2008\end{array}$ & $\begin{array}{l}\text { Radical Nefrectomia (2), } \\
\text { Pieloplastia desmembrada (3), } \\
\text { Nefrectomia parcial (1) }\end{array}$ & 2.0 & NP & 2.16 & R-Port & $\begin{array}{c}\text { Sangrado } \\
\text { postoperatorio }\end{array}$ \\
\hline $\begin{array}{l}\text { Raman et al, } \\
\text { Eur Urol, } 2008\end{array}$ & $\begin{array}{l}\text { Nefrectomia simple (5), } \\
\text { Radical Nefrectomia (6) }\end{array}$ & 2.0 & 20 & 2 & $\begin{array}{l}\text { Incisión } \\
\text { simple }\end{array}$ & Ninguna \\
\hline $\begin{array}{l}\text { Desai et al, } \\
\text { Urology, } 2008\end{array}$ & Prostatectomia simple (3) & 3.3 & 430 & 1.7 & R-port & Lesión intestinal \\
\hline $\begin{array}{l}\text { Ponsky et al, } \\
\text { J Endourol, } \\
2008\end{array}$ & Nefrectomia radical (1) & 1.5 & 10 & 2 & $\begin{array}{l}\text { Trocares } \\
\text { estándar } \\
\text { y sistema } \\
\text { Gelport }\end{array}$ & $\begin{array}{l}\text { Sin } \\
\text { complicaciones }\end{array}$ \\
\hline $\begin{array}{l}\text { Castelluci et al, } \\
\text { J Endourol, } \\
2008\end{array}$ & Adrenalectomia & 2 & 125 & 1 & Incisión simple & Ninguna \\
\hline $\begin{array}{l}\text { Msezane et al, } \\
\text { BJU Int, } 2008\end{array}$ & $\begin{array}{l}\text { Nefrectomia retroperitoneal } \\
(54)\end{array}$ & $52 \min (35-96)$ & $\begin{array}{l}\text { Reportado } \\
\text { como minimo }\end{array}$ & 1 & $\begin{array}{l}\text { Incisión simple } \\
\text { retroperitoneal }\end{array}$ & Ninguna \\
\hline $\begin{array}{l}\text { Milliken et al, } \\
\text { J Pediatr Urol, } \\
2008\end{array}$ & $\begin{array}{l}\text { Colocación de } \\
\text { catéteres } \\
\text { peritoneales (22) }\end{array}$ & $26 \min (23-29)$ & Ninguno & 1 & Incisión simple & $\begin{array}{l}\text { Fuga alrededor } \\
\text { del cateter, } \\
\text { infección } \\
\text { alrededor del } \\
\text { catéter, } \\
\text { obstrucción } \\
\text { del catéter. }\end{array}$ \\
\hline $\begin{array}{l}\text { Desai et al, } \\
\text { Urology, } 2008\end{array}$ & $\begin{array}{l}\text { Pieloplastia desmembrada (4), } \\
\text { Ureter ileal (1), } \\
\text { Ureteroneo cistostomia (1). }\end{array}$ & $\begin{array}{l}\text { PD:5.2, UI:5, } \\
\text { UNC: } 3\end{array}$ & $\begin{array}{l}\text { PD: } 75, \text { UI: } 75 \text {, } \\
\text { UNC: } 50\end{array}$ & $\begin{array}{l}\text { PD: } 1.5, \text { UI:3, } \\
\text { UNC: } 2\end{array}$ & R-port & Ninguna \\
\hline $\begin{array}{l}\text { Gill et al, } \\
\text { J Urol, } 2008\end{array}$ & Nefrectomía del donante (4) & 3.3 & 50 & 3.0 & R-port & Ninguna \\
\hline $\begin{array}{l}\text { Kaouk y Palmer, } \\
\text { BJU Int, } 2008\end{array}$ & Varicocelectomia (3) & $<1$ & Minimo & Ninguno & Uni X & Ninguna \\
\hline $\begin{array}{l}\text { Barret et el, } \\
\text { J Endourol, } \\
\text { In press }\end{array}$ & Prostatectomia radical (1) & 2.3 & $500 \mathrm{cc}$ & & $\begin{array}{l}\text { Incisión simple } \\
\text { y uso de robot. }\end{array}$ & Ninguna \\
\hline
\end{tabular}




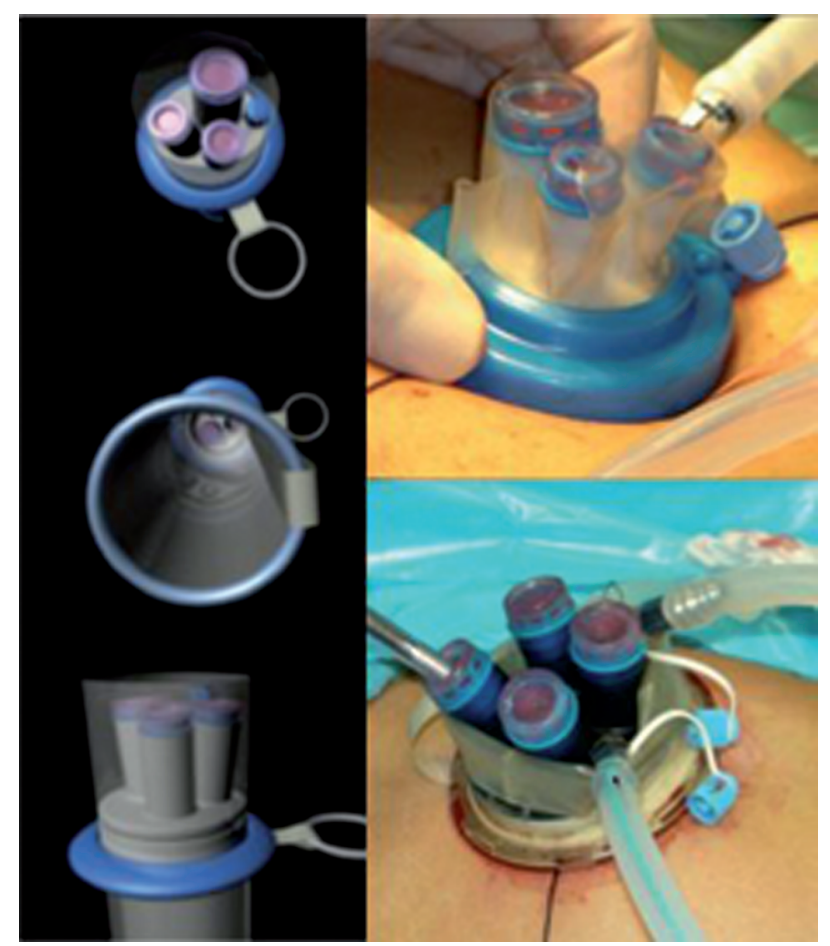

FIGURA 1. Fotos de los dos modelos de R-Port (Advanced Surgical Concepts, Dublin, Ireland).

(Cambridge Endo) y otros intencionalmente doblados de forma manual para la consecución de estos procedimientos (Tabla 2).

La posición del paciente se adopto de acuerdo al tipo de cirugía a realizar, así, la mayoría de los casos se colocaron de decúbito dorsal, excepto la paciente sometida a nefrectomía, quien fue posicionada de

Tabla 2. Equipos e Instrumentos básicos

1. R-port (Triport y Quadport. Advanced Surgical Concepts, Dublin, Ireland).

2. Instrumentos articulados flexibles de $5 \mathrm{~mm}$ : tijeras, porta agujas (Cambridge Endo).

3. Pinza de agarre descartable de $5 \mathrm{~mm}$ (manualmente doblado).

4. Porta-agujas laparoscópico recto (Olympus Medical, Tokyo, Japan)).

5. Cánula de succión - irrigación de 5mm (manualmente doblado).

6. Electrodo monopolar tipo gancho de $5 \mathrm{~mm}$ (manualmente doblado)

7. Tijera ultrasónica de $5 \mathrm{~mm}$ (SonoSurg, Olympus Medical, Tokyo, Japan)).

8. Pinza de Carter - Thomason.

9. Prostatotomo de Sotelo.

10. Bajanudos. decúbito lateral derecho. La vía de acceso fue transumbilical para todos los procedimientos menos las PSTV. Utilizando la técnica de abordaje abierta se realizó la colocación del dispositivo multicanal previamente.

\section{RESULTADOS}

La primera cirugía realizada fue una prostatectomía simple transumbilical transperitoneal, en un paciente de 67 años con APE en $5 \mathrm{ng} / \mathrm{ml}$ y biopsia de próstata con patología de hiperplasia benigna de próstata (HBP). El procedimiento fue técnicamente complicado en comparación con la laparoscopía estándar debido al poco espacio para manipular la óptica y la coordinación con los movimientos de los instrumentos los mismos que golpeaban entre si. Se accedió a la vejiga a través de una incisión transversa y con un punto de tracción en el adenoma el cual se exteriorizo con la ayuda de la pinza de Carter-Thomason, se procedió a la enucleación utilizando el electrodo monopolar tipo gancho de $5 \mathrm{~mm}$ previamente doblado en su tercio distal, tijeras articuladas, bisturí ultrasónico (SonoSurg, Olympus surgical, Tokyo, Japan) y una cánula de irrigaciónaspiración, también previamente doblada, que ayudó en la disección roma. Terminada la enucleación, se identificó la uretra que fue seccionada con las tijeras flexibles. Confirmada la hemostasia se colocó la sonda de Foley transuretral y se procedió a la cistorrafia en un plano, empezando en el lado derecho con un nudo deslizante (con un asa en el extremo), con puntos continuos hasta terminar en el extremo izquierdo donde se fijo la sutura con dos clips poliméricos de $5 \mathrm{~mm}$ (Hem-O-Lock, Weck Closure Systems, North Carolina, USA). A nivel pélvico, se dejo un dren Blake el que se exteriorizó por la cicatriz umbilical (Fig. 2).

El tiempo operatorio fue de 120 minutos y el sangrado de 200 cc. No hubo complicaciones intraoperatorias ni durante la hospitalización que duró 2 días. El dren fue removido a los 3 días y la sonda de Foley a la semana. El adenoma peso 95 gramos. La cicatriz quedó oculta en el ombligo y el paciente se encuentra continente con un control de uroflujo máximo en $78.2 \mathrm{ml} / \mathrm{seg}$ a los 6 meses de operado (Tabla 3).

El segundo caso, fue una paciente de 12 años con historia de infecciones urinarias a repetición. Entre los estudios realizados presentaba un gammagrama renal MAG3 que reporto función renal 


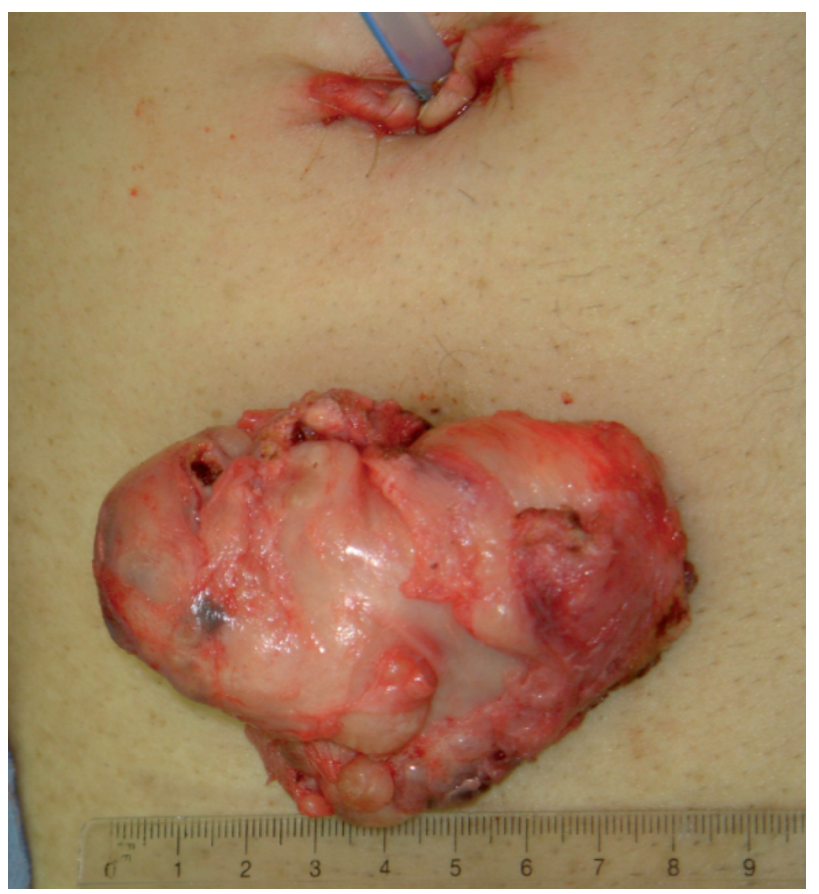

FIGURA 2. Foto de herida operatoria ubilical con dren y pieza operatoria.

izquierda de $4 \%$ y una tomografía axial computarizada que mostró importante hipotrofia renal izquierda. Reproduciendo los pasos reglados de la nefrectomía simple laparoscópica, se inició realizando la maniobra de Catell usando la tijera flexible que facilito la disección. Paralelamente se colocó un trocar de $2 \mathrm{~mm}$ para el uso de una pinza de 1,9 mm de diámetro. Se disecó el riñón y el uréter previa visualización del psoas. Identificados los vasos renales, se colocó clips poliméricos de $10 \mathrm{~mm}$ (Hem-O-Lock,
Weck Closure Systems, North Carolina, USA) y seccionó los mismos. Finalmente se completó la cirugía con ligadura y sección del uréter (Fig. 3).

El tiempo quirúrgico fue de 90 minutos, sangrado de 100 cc. y no presento complicaciones intra ni postoperatorias. La estancia hospitalaria fue de 2 días (Tabla 3). La cicatriz umbilical es prácticamente

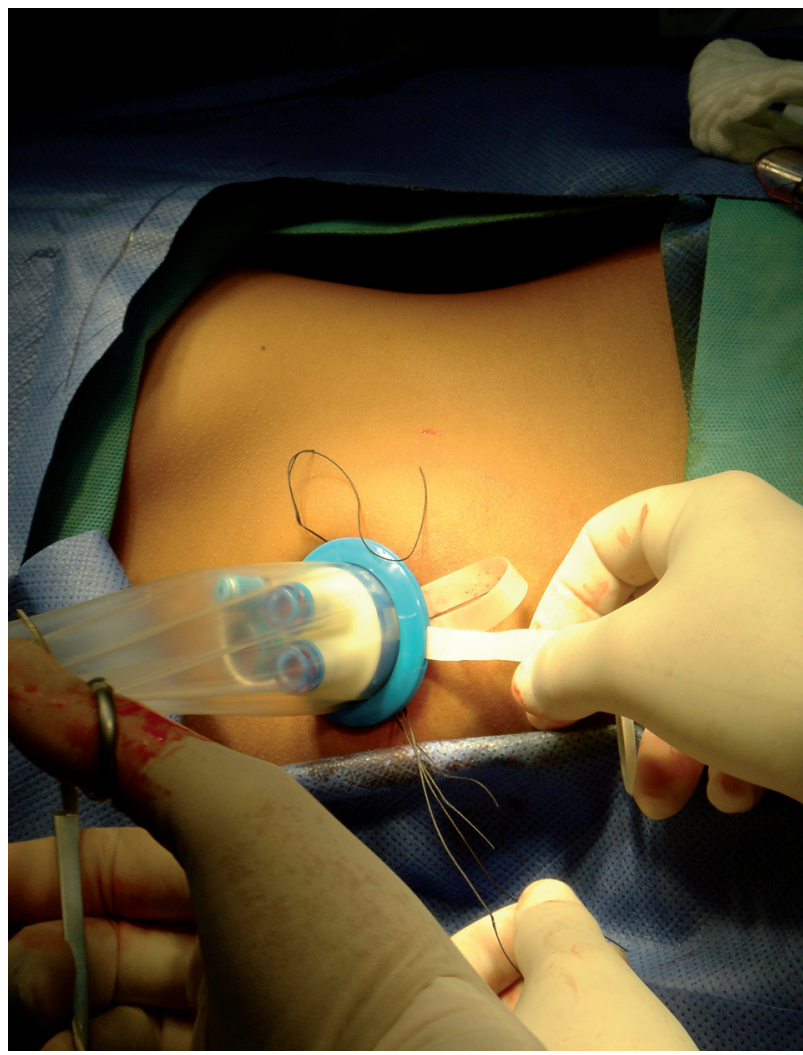

FIGURA 3. Colocación de TriPort en Nefrectomía simple.

Tabla 3. Datos perioperatorios y resultados. Prostatectomía simple transumbilical, Nefrectomía simple y Enterocistoplastia de aumento

\begin{tabular}{lccc}
\hline Cirugia & $\begin{array}{c}\text { Prostatectomia } \\
\text { simple transumbilical }\end{array}$ & $\begin{array}{c}\text { Nefrectomia simple } \\
\text { Enterocistoplastia } \\
\text { de aumento }\end{array}$ \\
\hline Edad (años) & 67 & 12 & 20 \\
Sexo & Masculino & Femenino & Femenino \\
ASA & 1 & 1 & 1 \\
Cirugía previa & No & No & No \\
Tiempo cirugía (minutos) & 120 & 90 & 300 \\
Sangrado (cc.) & 200 & 100 & 300 \\
Complicaciones & No & No & No \\
Sonda foley / drenes & SF/DPV & SF/D & SF/D \\
Estancia hospitalaria (días) & 2 & 1 & 7 \\
Días dren & 7 & 2 & 7 \\
Días sonda de Foley & Umbilical & 1 & 21 \\
Incisión localización & 2 & Umbilical & Umbilical \\
Incisión longitud (cm) & Hiperplasia & 2.5 & 6 \\
Anatomía patológica & & Displasia &
\end{tabular}


imperceptible. A inicios de mayo del presente año, en conjunto con el grupo de trabajo de Cleveland Clinic en Cleveland, Estados Unidos de América, Se consideró la alternativa de colocar el trocar directamente en la vejiga. Nuestra experiencia cooperativa alcanza hasta el momento 26 casos, en la presente publicación presentamos solo los procedimientos realizado en nuestro centro, correspondiente a 20 prostatectomías simples transvesicales (Fig. 4). El promedio de edad fue 68 años (rango 57-89 años), con un tiempo operatorio de 91 minutos (rango 45$210 \mathrm{~min}$ ), sangrado de 337 cc. (rango 50-1500 cc), dos casos cursaron con hematuria importante; el primero, a las 12 horas de la cirugía requirió exploración abierta para hemostasia y el segundo, a las 2 horas, exploración transuretral con hemostasia en áreas puntuales. Ambos pacientes evolucionaron sa-

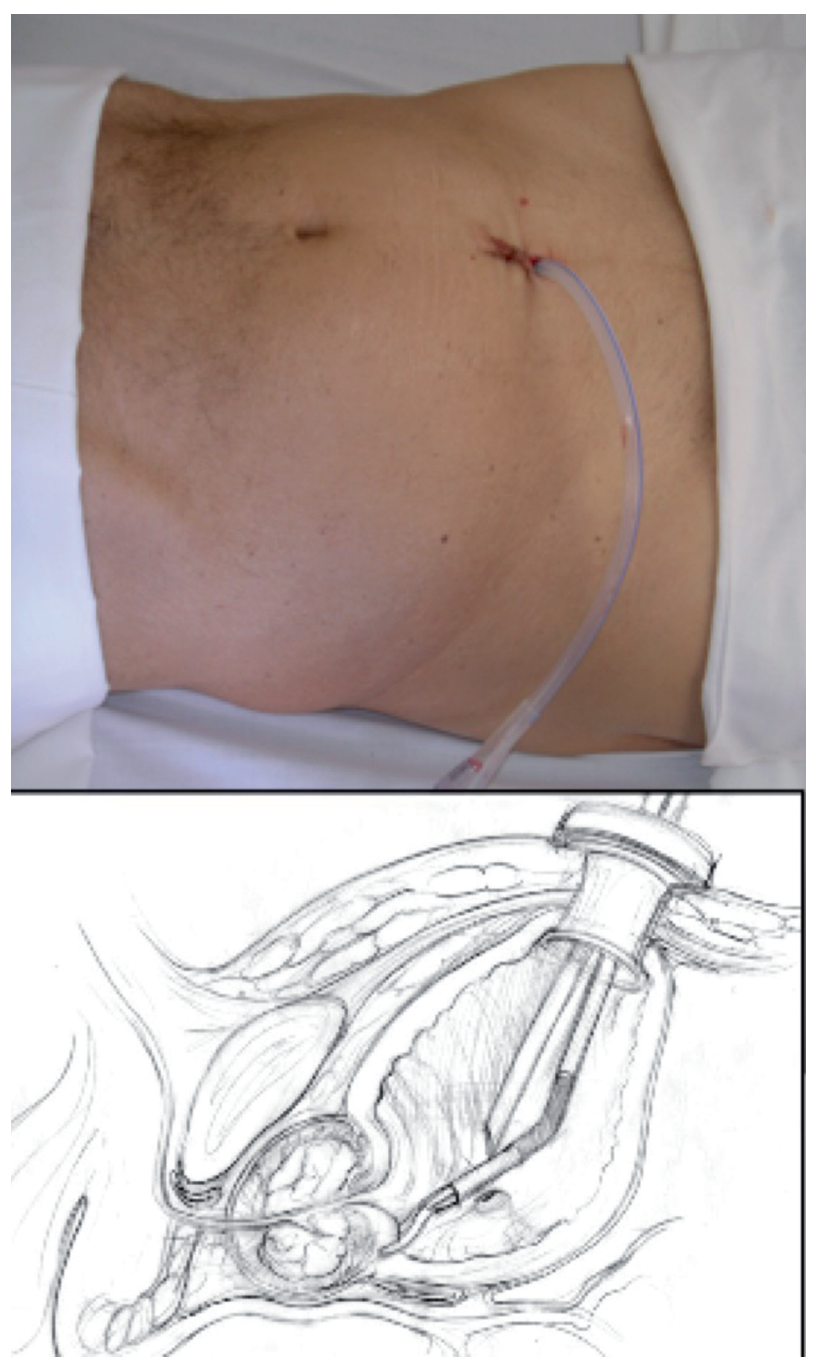

FIGURA 4. Foto de herida operatoria suprapúbica con catéter de cistostomía y esquema de instrumentos en cavidad vesical. tisfactoriamente. La estancia hospitalaria promedio fue de 1,8 días (rango 1-7 días) y el retiro de sonda de Foley fue en promedio a los 7.3 días (rango 5-12). El tamaño de la incisión no fue mayor de $3 \mathrm{~cm}$. El peso promedio de las piezas operatorias fue 56,7 gramos (rango 25 - 150 g) (Tabla 4).

Los resultados funcionales mostraron una evidente mejoría corroborada con cuestionarios como el IPSS, QoL (AUA) y en estudios urodinámicos (flujo máximo - Qmax), comparando los valores promedio preoperatorios: 21.6, 5.2 y 9.4; con los obtenidos un mes después de la cirugía: 3.9, 0 o 1 y 41.9 respectivamente (Tabla 5). Estéticamente la cicatriz infraumbilical fue minima, hasta la fecha no ha habido evidencia de hernias insicionales.

Tabla 4. Datos demográficos y perioperatorios. Prostatectomía simples transvesicales

\begin{tabular}{lcc}
\hline & Promedio & Rango \\
\hline Edad (años) & 68,3 & $57-89$ \\
Índice de masa corporal (IMC) & 24,64 & $23-28$ \\
APE basal (ng/ml) & 7,08 & $1,02-23,3$ \\
Síntomas preoperatorias (IPSS) & 21,6 & $15-33$ \\
Volumen x USTR (cc) & 88 & $50-247$ \\
Tiempo de cirugía (minutos) & 91,02 & $45-210$ \\
Sangrado (cc) & 337 & $50-1500$ \\
Complicaciones & 2 casos & sangrado \\
Días de hospitalización & 1,8 & $1-6$ \\
Días de sonda Foley & 7,3 & $5-12$ \\
Tamaño de la incisión (cm) & 2,55 & $2-3$ \\
Peso del adenoma (gms) & 56,7 & $25-140$ \\
\hline
\end{tabular}

$\mathrm{SF}$ : sonda de foley, C: cistectomía, D: dren prevesical.

Tabla 5. Resultados funcionales. Prostatectomías simples transvesicales

\begin{tabular}{lcc}
\hline & Promedio & Rango \\
\hline IPSS pre-operatorio & 21,6 & $15-33$ \\
IPSS post-operatorio & 3,9 & $1-5$ \\
QoL pre-operatorio & 5 ó 6 & \\
QoL post-operatorio & 0 ó 1 & \\
Qmax (ml/seg) pre-operatorio & 9,4 & $2-15$ \\
Qmax (ml/seg) post-operatorio & 41,9 & $32-84$ \\
Continencia pre-operatorio & Sí & \\
Continencia post-operatorio & Sí \\
\hline
\end{tabular}

SF: sonda de foley, C: cistectomía, D: dren prevesical. 
En coordinación con el Servicio de Ginecología de nuestra institución, se han realizado cinco histerectomías simples. Las indicaciones del procedimiento incluyeron carcinoma in situ con un margen positivo pos resección electroquirúrgica con asa $(\mathrm{N}=1)$, menorragia secundaria a miomatosis uterina $(\mathrm{N}=3)$ y menometrorragia con anemia $(\mathrm{N}=1)$. La histerectomía simple fue realizada siguiendo los pasos reglados de la cirugía laparoscópica. Con ayuda de un manipulador uterino, se disecaron las arterias uterinas para ligarlas con clips poliméricos o Ligasure, el resto de la disección se realizo utilizando bisturí ultrasónico y tijeras articulables (Cambridge Endo), además de pinzas de tracción dobladas previamente. La pieza se extrajo por vagina y el cierre de la cúpula fue realizado transvaginal (Fig. 5).

La edad promedio fue 46.4 años (rango 41-54 años). El tiempo operatorio fue 112 minutos (rango 90-160 min). El sangrado fue 118 cc. (rango 100160). No hubo complicaciones intraoperatorias ni postoperatorias. La estancia hospitalaria fue de 24 horas en los casos 1, 2, 4 y 5, y 36 horas en el caso 3. La patología final reveló Carcinoma in situ con extensión glandular (caso 1), miomatosis uterina (casos 2, 4 y 5), adenomiosis y endometritis (caso 3) (Tabla 6).

De la misma forma, fue intervenida quirúrgicamente una paciente de sexo femenino de 20 años con antecedente de lipoma sacro congénito operado a los 2 años de edad y sintomatología de larga data caracterizada por incontinencia, frecuencia, urgen-

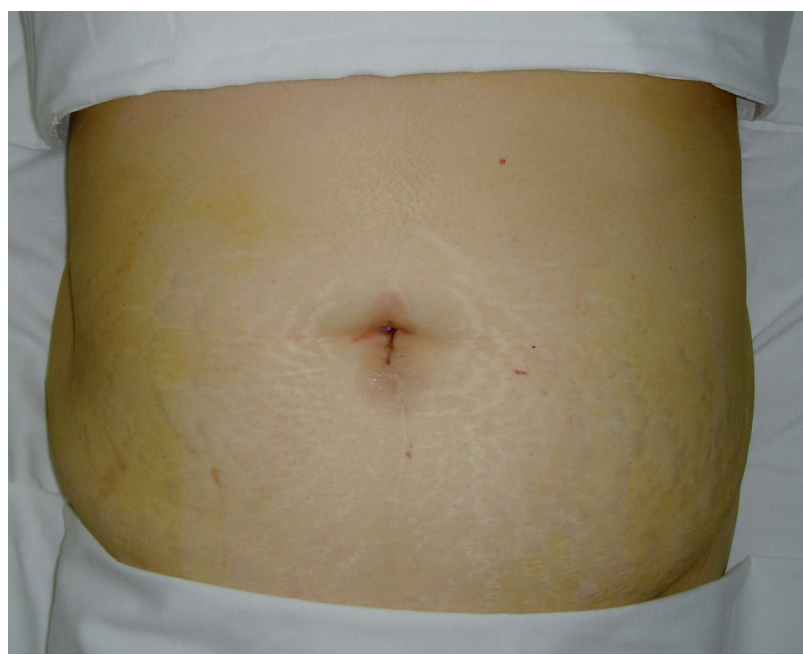

FIGURA 5. Foto de resultado cosmético con herida operatoria transumbilical mínima.

cia e infecciones urinarias a repetición en relación a vejiga neurogénica. Los estudios imagenológicos y funcionales (urodinamia) corroboraron el diagnostico encontrando importante disminución de tamaño vesical con paredes engrosadas, múltiples divertículos, inestabilidad del detrusor con volúmenes de 30 a 50 cc. y capacidad vesical disminuida (máximo $100 \mathrm{cc})$

La enterocistoplastía de aumento fue realizada a través del dispositivo de cuatro canales (QuadPort, Advanced Surgical Concepts, Dublin, Ireland), colocado transumbilical. Con la ayuda visual de la óptica flexible de $5 \mathrm{~mm}$ (EndoEye, Olympus Medical, Tokyo, Japan), el SonoSurge (Olympus Medical, Tokyo,

Tabla 6. Datos demográficos y perioperatorios. Histerectomías simples

\begin{tabular}{|c|c|c|c|c|c|c|}
\hline Hiterectomía simple & Caso 1 & Caso 2 & Caso 3 & Caso 4 & Caso 5 & Promedio \\
\hline Fecha & 04-abril & 20-junio & 26-julio & 24-agosto & 23-septiembre & \\
\hline Edad (años) & 47 & 54 & 41 & 48 & 42 & 46,4 \\
\hline ASA & 1 & 2 & 1 & 1 & 1 & 1,2 \\
\hline Tiempo cirugía (min) & 120 & 160 & 90 & 100 & 90 & 112 \\
\hline Sangrado (cc) & 160 & 110 & 100 & 100 & 120 & 118 \\
\hline Complicaciones & No & No & No & No & No & No \\
\hline Estancia hospitalaria (horas) & 24 & 24 & 36 & 24 & 24 & 26,4 \\
\hline Incisión localización & Umbilical & Umbilical & Umbilical & Umbilical & Umbilical & Umbilical \\
\hline Tamaño (cm) & 2,5 & 2,5 & 2,5 & 2,5 & 2,5 & 2,5 \\
\hline $\begin{array}{l}\text { Pieza operatoria (gramos/ } \\
\text { comparación sem. gestación) }\end{array}$ & $\begin{array}{l}250 \mathrm{~g} / \\
10 \mathrm{sem}\end{array}$ & $\begin{array}{l}300 \mathrm{~g} / \\
12 \mathrm{sem}\end{array}$ & $\begin{array}{l}350 \mathrm{~g} / \\
13 \mathrm{sem}\end{array}$ & $\begin{array}{l}280 \mathrm{~g} / \\
12 \mathrm{sem}\end{array}$ & $\begin{array}{l}200 \mathrm{~g} / \\
9 \mathrm{sem}\end{array}$ & \\
\hline Anatomía patológica & $\begin{array}{l}\text { Carcinoma } \\
\text { in situ }\end{array}$ & $\begin{array}{c}\text { Miomatosis } \\
\text { uterina }\end{array}$ & $\begin{array}{l}\text { Aenomiosis } \\
\text { endometritis }\end{array}$ & $\begin{array}{c}\text { Miomatosis } \\
\text { uterina }\end{array}$ & $\begin{array}{l}\text { Miomatosis } \\
\text { uterina }\end{array}$ & \\
\hline
\end{tabular}


Japan), la tijera flexible (Cambridge Endo) conectada al monopolar en corte y la pinza tractora, se realizó la cistectomía parcial resecándose el $70 \%$ del órgano hueco respetándose el trígono vesical. Culminada la etapa ablativa y revisada la hemostasia, se identificó un segmento de $60 \mathrm{~cm}$ de íleon, seccionando su meso a 15 y $75 \mathrm{~cm}$ de la válvula ileocecal con SonoSurge. Luego, retirado el inserto valvular central del trocar transumbilical y de forma extracorpórea se completó la sección del intestino, reconstituyó el tránsito intestinal con anastomosis ileo-ileal latero-lateral con sutura automática y finalmente se conformó la bolsa ileal manualmente con sutura PDS 2-0. Se introdujo el pouch preparado para la ampliación de vejiga y se recolocó la parte valvular del trocar restableciendo el neumoperitoneo. Laparoscópicamente se verifico la orientación adecuada del pouch intestinal y se completo la anastomosis vesico-ileal con varios segmentos de sutura continua con PDS 2-0 (Fig. 6).

El tiempo operatorio fue de 300 minutos con sangrado de 300cc. No hubo complicaciones intraoperatorias ni postoperatorias. La estancia hospitalaria fue de 6 días. El retiro de dren y sonda de Foley se realizó a los 7 y 21 días de la cirugía respectivamente (Tabla 3). Ha presentado evidente mejoría funcional al tener mayor capacidad vesical corroborada mediante neocistografía. El resultado cosmético fue adecuado con una cicatriz de $5 \mathrm{~cm}$.

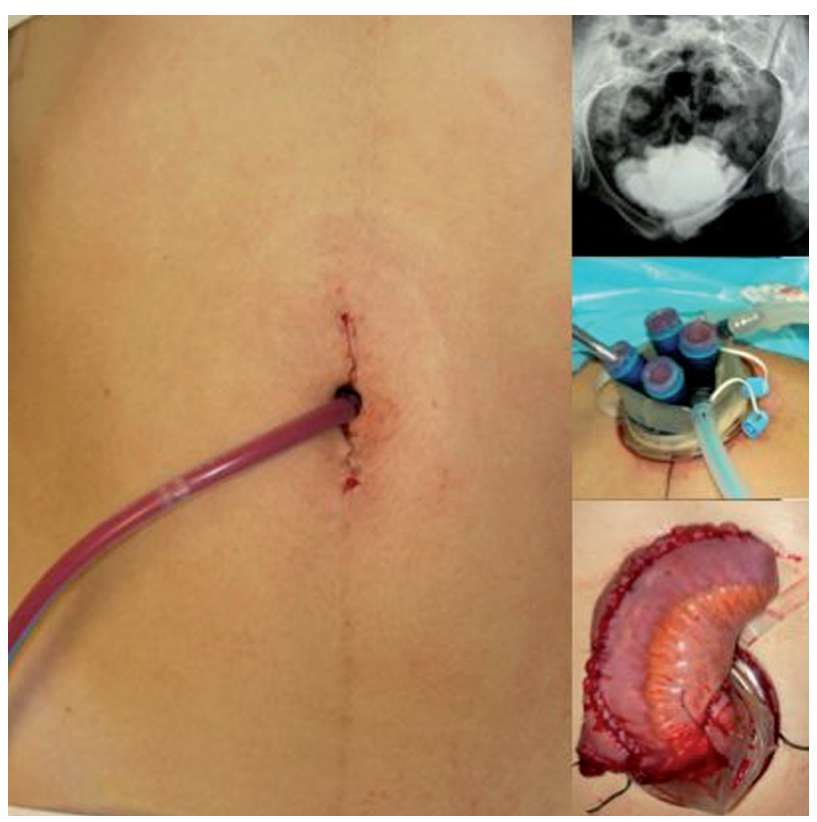

FIGURA 6. Fotos de herida operatoria transumbilical con dren. A la derecha de arriba abajo. Cistografia preoperatoria, neovejiga y Quadport desplegado.

\section{DISCUSIÓN}

La cirugía laparoscópica presenta, frente a la cirugía abierta, una clara disminución del dolor postoperatorio, una menor estancia hospitalaria y una menor convalecencia. Asimismo, una reducción significativa de la aparición de adherencias intraperitoneales postoperatorias respecto a la cirugía abierta, lo que constituye un factor importante en la elección de esta técnica ${ }^{8,9}$. Si evaluamos la cirugía laparoscópica estándar frente a la realizada a través de un solo trocar, encontraremos desventajas como el uso de varias incisiones para la introducción de los trocares con el consiguiente trauma del acceso quirúrgico puesto en evidencia por dolor, espasmos musculares y no infrecuentes lesiones de los vasos epigástricos ${ }^{10}$. Si bien LESS tiene ventajas en cuanto al menor trauma producido por los trocares y resultados cosméticos superiores, también tiene el inconveniente de una curva de aprendizaje más larga por la dificultad técnicas en la actualidad.

Asumimos que al igual que otros grupos que emprendieron este camino con esta nueva alternativa a las técnicas quirúrgicas laparoscópicas convencionales, empezamos con procedimientos de resección como la histerectomía simple o la nefrectomía en un riñón hipotrófico, utilizando los instrumentos laparoscópicos habituales disponibles y adaptando otros como la cánula de irrigación-aspiración, una pinza retractora y el electrodo monopolar tipo gancho de $5 \mathrm{~mm}$, que fueron manualmente doblados. Luego, con el desarrollo de instrumentos flexibles, utilizados con la mano más hábil inicialmente y apoyándose en un instrumento rígido en la otra mano se fue adquiriendo las destrezas necesarias para la manipulación de las mismas y por ende, la incursión en procedimientos reconstructivos como la prostatectomía simple transvesical y la enterocistoplastía de aumento ${ }^{11,12}$.

Actualmente se están buscando mejoras en los equipos utilizados, desde nuestro punto de vista, el dispositivo multicanal que mejor se adapta o se acerca más al concepto de "ideal" es el R-Port (Advanced Surgical Concepts, Dublin, Ireland) con sus dos modelos disponibles, el TriPort y el GuadPort que cuentan con una válvula con canales independientes para el ingreso del CO2, la óptica y las pinzas ${ }^{20-21}$. Es importante recordar que al colocar el dispositivo en la pared abdominal, para mantenerlo fijo y evitar se movilice durante la cirugía, por su diámetro, no se deben realizar incisiones en la aponeurosis mayores de 2,5 ó $6 \mathrm{~cm}$, dependiendo del modelo usado. 
Aun no disponemos de instrumentos articulados "ideales" con una o más curvas, que permitan restablecer internamente el principio de triangulación y externamente eviten la colisión de las manos. Estos nuevos conceptos, aun en prototipos, son de difícil construcción considerando que las pinzas deben roticular en su eje, alinear la punta con su eje en relación a los puntos de flexión, además de la dificultad de hacerlos desarmables para poder esterilizarlos. El portaagujas articulable (Cambridge Endo), que viene siendo mejorado progresivamente, tiene dentro de sus fortalezas el mecanismo de fijación que permite mantener el instrumento en una posición, la excelente articulación y roticulación, sin embargo también tiene debilidades como son la poca firmeza al momento de asegurar los nudos y lo voluminoso del mango de trabajo, razones por la que aun en muchos de los procedimientos reconstructivos se usa el portaagujas convencional en la mano dominante y/o un trocar adicional de $2 \mathrm{~mm}$. a través del que se introduce una pinza de $1.9 \mathrm{~mm}$ que permite ayudar en la síntesis, ya que es muy difícil anudar con dos instrumentos rígidos paralelos entre si. Los nuevos prototipos de estos instrumentos articulables prometen aumentar la firmeza del portaagujas y reducir el tamaño del mango mejorando la ergonomía.

De acuerdo al consenso LESSCAR (LaparoEndoscopic Single-Site Surgery Consortium for Assessment and Research) celebrado en Cleveland, Ohio el 07 de julio del año 2008, se estima que el volumen de procedimientos aplicables a LESS puede aumentar en aproximadamente un 50\% durante los próximos cinco años, con un porcentaje aún mayor en algunas especialidades en las que puede llegar hasta $80 \% 13,14$.

Es importante conocer que los progresivos intentos quirúrgicos a la par de los desarrollos tecnológicos, determinaran que tipos de procedimientos se podrán ir haciendo a través de un trocar único, en forma segura, sin que se requiera de habilidades laparoscópicas excepcionales, de lo contrario no se lograra la difusión de la técnica. En general, los procedimientos reconstructivos son los de mayor grado de dificultad y que requieren de gran habilidad y conocimiento previo del manejo laparoscópico de dicha técnica. Los procedimientos realizados en nuestra experiencia inicial son una muestra de la aplicabilidad de este nuevo concepto de cirugía mínimamente invasiva, en esta ocasión con el uso del dispositivo multicanal (R-Port, Advanced Surgical Concepts, Dublin, Ireland) y los diversos instrumentos flexibles y otros adaptados para la cirugía.

Debemos fundamentalmente comparar los resultados de nuestra prostatectomía transvesicales LESS con la prostatectomía simple por laparoscopia con o sin robot, sin embargo estas comparaciones no serían objetivas en este momento. Fundamentalmente debemos avocarnos a registrar los detalles más sutiles, síntomas irritativos y recuperación, además de mantener en mente la idea de que deben establecerse protocolos prospectivos de evaluación buscado verificar los detalles más precisos que ofrezcan la evidencia necesaria para sustentar definitivamente la técnica. La indicación de adenomectomia simple en LESS es evidentemente controversial si la comparamos con el estándar de oro para el tratamiento de la hiperplasia benigna de la próstata, pero el abordaje por trocar único se encuentra evolucionando y esta técnica es presentada como una innovación que pudiese incorporarse se manera efectiva en el armamentario del cirujano.

Con relación a la colocación de extra-trocares en la realización de LESS, consideramos que es totalmente justificada para garantizar la seguridad del paciente, que es nuestro fin último. La colocación del trocar adicional en la nefrectomía realizada en la presente serie no es observada por nuestro grupo como "conversión" pues la técnica de mínimo acceso se mantuvo en todo momento. Nuestra recomendación inicial es considerar la utilización de extratrocares, siempre que el cirujano lo estime necesario, especialmente en los inicios de su experiencia con LESS. La experiencia acumulada hasta hoy en LESS es limitada y representa el inicio de un campo en plena expansión, especialmente en la Urología.

En términos de estudios comparativos existen en la actualidad dos estudios realizados entre la técnica con trocar único y laparoscopia convencional en nefrectomía (simple y radical) y nefrectomía para el donante vivo ${ }^{26-27}$. Los resultados de ambos estudios son prometedores y aunque se trata de series cortas, estos manuscritos abren el camino a seguir en esta nueva técnica.

La presente serie, bien pudiese ser considerada heterogénea en el sentido estricto de la evaluación quirúrgica, sin embargo representa una experiencia pionera a nivel mundial y es nuestro objetivo presentarla a la comunidad urológica con la finalidad de expandir las fronteras de la cirugía de mínimo acceso. 


\section{CONCLUSIONES}

La cirugía laparoscópica a través de un solo trocar aplicada a procedimientos como los presentados en esta revisión, es una alternativa factible y reproducible. Sin embargo faltan aún mayores estudios, experiencias y el desarrollo de nuevas tecnologías que faciliten más la consecución de estos procedimientos.

\section{REFERENCIAS}

1. Rattner D, Kalloo A and the SAGES/ASAGE working group on natural orifice translumenal endoscopic surgery. Surg Endosc 2006;20(2):329-333.

2. Rattner D and Hawes R. NOTES: Gathering momentum. Gastrointest Endosc 2006;63(6): 838-39.

3. Pelosi MA: Single puncture advance operative laparoscopy. International Congress of Gynecologic Endoscopy. The American Association of Gynecologic laparoscopists 19th Annual Meeting November 1990, Orlando, Florida.

4. Pelosi MA, Pelosi MAIII, Laparoscopic hysterectomy with bilateral salpingo-ooforectomy using a single umbilical port. NJ Med 1991; 88(10): 721-726.

5. Esposito C. One-trocar appendectomy in pediatric surgery. Surg Endosc 1998;12(2):177-178.

6. Ates O, Hakguder G, Olguner M, Akgur FM. Single port laparoscopic appendectomy conducted intracorporeally with the aid of a transabdominal sling suture. J Pediatr Surg 2007;42 (6):10711074.

7. Raman JD, Bensalah K, Bagrodia A, Stern JM, Cadeddu JA. Laboratory and clinical development of single keyhole umbilical nephrectomy. Urology. 2007;70(6):1039-1042.

8. Isariyawongse JP, McGee MF, Rosen MJ, Cherullo EE and Ponsky LE. Pure natural orifice transluminal endoscopic surgery (NOTES) nephrectomy using instruments in the porcine model. J Endourol 2008;22(5):1087-1091

9. Castellucci SA, Curcillo PG, Ginsberg PC, Saba SC, Jaffe JS and Harmon JD. Single port acces adrenalectomy. J Endourol 2008;22(8):1573-1576.

10. Gill IS, Canes D, Aron M, Haber GP, Goldfarb DA, Flechner S, et al. Single port transumbilical (E-NOTES) donor nephrectomy. J Urol 2008;180(2):637-641.

11. Kaouk JH, Haber GP, Goel RK, Desai MM, Aron M, Rackley RR, et al. Single-port laparoscopic surgery in urology: initial experience. Urology. 2008;71:3-6.

12. Desai MM, Rao PP, Aron M, Pascal-Haber G, Desai MR, Mishra $\mathrm{S}$, et al. Scarless single port transumbilical nephrectomy and pyeloplasty: first clinical report. BJU Int. 2008;101(1):83-88

13. Gill IS and LESS Consortium. Consensus statement on the Consortium for Laparoendoscopic Single Site (LESS) Surgery. Ann Surg (En Prensa).

14. Box G, Averch T, Badlani G, Cadeddu J, Cherullo E, Clayman R, et al. Nomenclature of Natural Orifice Translumenal Endoscopic Surgery (NOTES) and Laparo-Endoscopic Single-Site Surgery (LESS) in Procedures in Urology. J Endourol. 2008;22 (11):2575-2581.
15. Bucher P, Pugin F and Morel P. Single Port Acces laparoscopic right hemicolectomy. Int J Colorectal Dis 2008; 23(10):10131016.

16. Desai M, Aron M, Stein R, Rao P, Canes D, Aron M, et al. Embrionic Natural Orifices Transumbilical Endoscopic Surgery (E-NOTES) for advanced reconstruction: Initial experience. Urology 2008. (En Prensa) .

17. Piskun G, and Rajpal S. Transumbilical laparoscopic cholecystectomy utilizes no incisions outside the umbilicus. J Laparoendosc Adv Surg Tech 1999; 9(4): 361-364.

18. Kaouk JH and Palmer JS. Single-port laparoscopic surgery: initial experience in children for varicocelectomy. BJU Int. 2008; 102(1):97-99.

19. Desai MM, Monish A, Canes D, Fareed K, Carmona O, Haber GP, et al. Single-port transvesical simple prostatectomy: Initial clinical report. Urology 2008;72(5):960-965.

20. Rane A, Rao PP, Rao SP, Bonadio F, Rao PP. Clinical evaluation of a novel laparoscopic port (R-PortTM) in urology and evolution of the single laparoscopic port procedure (SLAPP). J Endourol 2007; Abstract BR6-01.

21. Rane A, Rao P and Rao P. Single Port Acces nephrectomy and other laparoscopic urologic procedures using a novel laparoscopic port (R-port). Urology 2008; 72 (2): 260-263.

22. Nguyen NT, Reavis KM, Hinojosa MW, Smith BR and Wilson SE. Laparoscopic transumbilical cholecystectomy whitout visible abdominal scar. J Gastrointest Surg. 2008 Aug 15. (

23. Remzi FH, Kirat HT, Kaouk JH and Geisler DP. Single port laparoscopy in colorectal surgery. Colorectal Dis 2008;10(8): 823-826.

24. Canes D, Desai MM, Aron M, Haber GP, Goel RK, Stein RJ, et al. Transumbilical Single-Port Surgery: Evolution and Current Status. Eur Urol. 2008;54 5):1020-1029.

25. Desai M, Aron M, Berger A, Canes D, Stein R, Haber G-P, et al Transvesical robotic radical prostatectomy. BJU Int 2008;102 (11): 1666-1669.

26. Raman JD, Bagrodia A, Cadeddu JA. Single-Incision, Umbilical Laparoscopic versus Conventional Laparoscopic Nephrectomy: A Comparison of Perioperative Outcomes and Short-Term Measures of Convalescence. Eur Urol. 2008 Aug 13 (En Prensa).

27. Canes D, Berger A, Aron M, Haber GP, Kaouk J, Goldfarb D , et al. Single Port versus standard laparoscopic donor nephrectomy: matched-pair comparison. J Urol 2008 (En Prensa).

Correspondencia autor: Dr. Rene Sotelo Noguera Centro de Cirugía Robótica y Mínimamente Invasiva, Instituto Médico La Floresta,

Av. Principal Urb. La Floresta, PB 707.

Caracas, Venezuela.

Tel.: +58 212209 6240; 2851015 .

E-mail autor: renesotelo@cantv.net, renesotelo@mac.com

Información artículo: Original - Laparoscopia

Trabajo recibido: diciembre 2008

Trabajo aceptado: enero 2008 